\title{
La microbiota intestinal: Un nuevo actor en el desarrollo de la obesidad
}

\author{
PAMELA MORALES ${ }^{\text {la }}$, JERUSA BRIGNARDELLO $^{\text {bb }}$, MARTÍN GOTTELAND $^{\text {1c }}$
}

'Laboratorio de Microbiología y Probióticos, Instituto de Nutrición y Tecnología de los Alimentos (INTA), Universidad de Chile, Santiago, Chile.

Trabajo financiado por proyecto Fondecyt \# 1080519

Recibido el 28 de enero de 2010, aceptado el 28 de mayo de 2010.

aBioquímico, Estudiante del Programa de Doctorado en

Nutrición y Ciencias de los Alimentos de la Universidad de Chile.

${ }^{\mathrm{b}}$ Nutricionista, Magister en Nutrición (INTA) ${ }^{\mathrm{C}} \mathrm{PhD}$ en Fisiología y Fisiopatología de la Nutrición Humana.

Correspondencia a: Martín Gotteland INTA, Universidad de Chile. El Libano 5524, Macul Santiago, Chile. F: $56-2-9781471$

Fax: 56-2-2214030 E-mail: mgottela@inta.cl

\section{The association of intestinal microbiota with obesity}

Intestinal microbiota (IM) plays a role in the development of obesity and its associated low-grade inflammation. Bacterial colonization of the gastrointestinal tract of germ-free mice (without microbiota) increases by $60 \%$ their fat mass, alters their fasting glucose and insulin levels, triples their hepatic triglycerides and induces adipocyte hypertrophy. IM favors fat storage in adipocytes through the inhibition of Fiaf (Fasting-Induced Adipocyte Factor), an inhibitor of lipoprotein lipase. Compared with normal weight subjects, the IM from obese exhibits a higher proportion of Firmicutes/Bacteroidetes and is more efficient in extracting energy from foodstuffs. The loss of bodyweight by a hypocaloric diet reverts the proportion of bacteria to that of lean subjects. The intake of a high fat diet also alters the IM, affecting intestinal barrier function and favoring endotoxinemia. These events increase oxidative and pro-inflammatory processes in plasma and peripheral tissues and increment the risk of insulin resistance. Such events are reverted by the administration of prebiotics which stimulate the growth of Bifidobacterium and Lactobacillus species in the colon, reestablishing the gut homeostasis. Interestingly, products resulting from the fermentation of prebiotics stimulate the differentiation of enteroendocrine cells and the release of glucagon-like peptide 1 and peptide YY, that have insulin-like and anorexigenic activities, thus contributing to body weight equilibrium.

(Rev Med Chile 2010; 138: 1020-1027).

Key words: Insulin resistance; Obesity; Prebiotics.
$\mathrm{E}$ 1 tubo digestivo (TD) alberga un ecosistema bacteriano complejo cercano a los 100 trillones $\left(10^{14}\right)$ de microorganismos que forman la microbiota intestinal (MI). Esta MI ha evolucionado junto con el ser humano, adaptándose y conviviendo con él en una estrecha relación simbiótica: la microbiota ejerce funciones nutricionales, metabólicas y protectoras que la vuelven indispensable para el huésped mientras que este le entrega nutrientes y condiciones adecuadas para su crecimiento. La presencia de la MI, de hecho, impacta fuertemente en la expresión de genes en la mucosa intestinal del huésped: por ejemplo la colonización de ratones axénicos (sin microbiota) por Bacteroides thetaiotaomicron (un microorganismo común en la MI) induce la expre- sión de genes implicados tanto en la defensa del organismo y la regulación de la función intestinal de barrera como en la vascularización del epitelio y la digestión/absorción de nutrientes ${ }^{1}$. La MI no se distribuye en forma homogénea en el TD; más de $99,9 \%$ se encuentra en el colon, con concentraciones que alcanzan los $10^{9}$ a $10^{11} \mathrm{CFU} / \mathrm{g}$ y con un fuerte predominio de anaerobios estrictos. El ciego y el colon ascendente son sede de procesos intensos de fermentación y sus poblaciones bacterianas están en continuo crecimiento, produciendo concentraciones altas de ácidos grasos volátiles (AGV) que llevan a un $\mathrm{pH}$ bajo $(5,4-5,9)$, mientras que en el colon descendente estos procesos son menos intensos por lo cual la concentración de AGV en su lumen es menor y el pH más alto 
$(6,6-6,9)^{2}$. En la MI existe un equilibrio entre las poblaciones bacterianas dominantes (Bacteroides, Clostridium, Eubacterium) y aquellas subdominantes, presentes en menor cantidad. Cuando este equilibrio es adecuado, la microbiota actúa como una barrera que impide la multiplicación de patógenos y el desarrollo de patologías gastrointestinales. Factores cotidianos tales como el estrés o el consumo de agua clorada afectan a la MI, pero dichas alteraciones son menores comparadas con aquellas producidas por el consumo regular de anti-inflamatorios, laxantes o antiácidos, o la aplicación de radio -o quimioterapia ${ }^{3}$. La administración de antibióticos impacta en forma considerable el equilibrio de la $\mathrm{MI}^{4}$, reduciendo drásticamente las poblaciones dominantes y favoreciendo la emergencia de patógenos oportunistas como Clostridium difficile 5 . Por otra parte, perturbaciones cuali- y/o cuantitativas de la MI han sido observados en un gran número de patologías que afectan directamente el TD (enfermedades inflamatorias crónicas del intestino, cáncer colo-rectal, constipación, diarreas) o a distancia de él (alergias, artritis $)^{6}$. Aún si no está claramente determinado si estas alteraciones de la MI son causas o consecuencias de dichas patologías, cabe destacar que están frecuentemente asociadas con alteraciones de la función intestinal de barrera y del sistema inmune asociado con la mucosa? ${ }^{7}$.

La mejor comprensión de la composición y funcionamiento de la MI ha permitido el desarrollo de los conceptos de prebióticos y probióticos. Los primeros son principalmente hidratos de carbono no-digestibles (fibras dietéticas solubles) cuya fermentación en el colon estimula el crecimiento de microorganismos (bifidobacteria y lactobacilos principalmente) beneficiosos para la salud del huésped mientras que los segundos son microorganismos inocuos que pueden sobrevivir su pasaje por el TD, donde ejercen actividades saludables ${ }^{8}$. Tanto los prebióticos como los probióticos pueden ser considerados como herramientas útiles para mantener el equilibrio armonioso de la MI a través del manejo de la dieta del individuo.

\section{El rescate de energía por la MI}

La principal manifestación del mutualismo entre la MI y su huésped es que la primera es capaz de extraer energía provenientes del almi- dón resistente y de los poli- y oligosacáridos que forman la mayoría de la fibra dietética soluble, haciéndola disponible para el huésped y evitando de esta forma su perdida en las deposiciones. $B$. tethaiotaomicron, por ejemplo, puede expresar 175 hidrolasas distintas, lo que le otorga plasticidad para adaptarse tanto a los diversos polisacáridos de la dieta como a los endógenos presentes en el glicocalix del ribete estriado y en las mucinas? La fermentación de estos sustratos libera agua, gases $\left(\mathrm{CO}_{2}, \mathrm{H}_{2}, \mathrm{CH}_{4}\right)$ y AGV, principalmente acetato, propionato y butirato en una proporción aproximada de $60 \%, 20 \%, 20 \%$, dependiendo de la naturaleza de la fibra; estos AGV son absorbidos y oxidados, permitiendo el salvataje de energía ${ }^{10}$. La importancia de este fenómeno es ilustrada por los ratones axénicos que, al carecer de MI, deben consumir 20 a 30\% más de alimento que los animales convencionales (con microbiota) para lograr crecimientos comparables ${ }^{11}$. En el ser humano se estima que alrededor de 7 a 10\% de las calorías absorbidas provienen diariamente de este proceso ${ }^{12}$. Mientras que el butirato es metabolizado principalmente por los colonocitos, el acetato y el propionato son absorbidos, alcanzando concentraciones de 300 a $450 \mu \mathrm{M}$ en la sangre portal y de 50 y $100 \mu \mathrm{M}$ en la sangre periférica ${ }^{2}$. Una vez captado por el hígado, el acetato sirve de substrato preferencial para la gluconeogénesis y la síntesis de colesterol y de triglicéridos, mientras que el propionato inhibe la expresión génica de las enzimas hepáticas involucradas en esta lipogénesis de novo ${ }^{10}$.

El reconocimiento del rescate colónico como una fuente regular y significativa de calorías para el organismo hace que en la actualidad las instancias responsables (Codex) están discutiendo la posibilidad de atribuir un cierto valor energético $(2 \mathrm{Kcal} / \mathrm{g})$ a la fibra soluble presente en alimentos.

\section{Rol de los AGV en el control del apetito}

Cani et al, fueron los primeros en mostrar que la administración a ratas de una dieta con $10 \%$ oligofructosa disminuía el consumo de alimentos y la masa grasa epididimal después de 3 semanas. Los autores atribuyeron este hallazgo a la menor liberación de grelina, una hormona orexigénica producida por las células enteroendocrinas de la mucosa gástrica, y a la mayor liberación de GLP1 y PYY, hormonas anorexigénicas producidas 
por las células enteroendocrinas L de la mucosa ileal y colónica ${ }^{13}$. Posteriormente mostraron que productos de la fermentación de la oligofructosa, probablemente los AGV, aumentan el número de células enteroendocrinas L en la mucosa del colon proximal, mediante la inducción de los factores de diferenciación neurogenina-3 y NeuroD ${ }^{14}$. El consumo de este prebiótico permitió mejorar también la glicemia e insulinemia en ayuno de ratas diabéticas e inhibir el apetito de animales alimentados con una dieta alta en grasa ${ }^{13,15}$. Estimulados por estos hallazgos, estos autores desarrollaron un ensayo clínico randomizado, en doble-ciego y controlado en 10 voluntarios sanos que recibieron diariamente $16 \mathrm{~g}$ de prebiótico o de maltodextrina por 2 semanas. El consumo de oligofructosa aumentó la fermentación en el colón (evaluada mediante test de $\mathrm{H}_{2}$ en aire espirado) y, en forma correlativa, las concentraciones plasmáticas de GLP-1 y de PYY. Estos efectos de la oligofructosa podrían explicar la disminución de la sensación de hambre y la baja post-prandial de la glicemia, observadas en estos sujetos ${ }^{16}$. Resultados similares fueron descritos por Parnell et al, en un ensayo clínico realizado en 48 adultos sanos que recibieron diariamente $21 \mathrm{~g}$ de oligofructosa o de maltodextrina por 12 semanas; no-obstante, a diferencia del estudio anterior, los autores no observaron cambios del GLP-1 plasmático pero detectaron una menor concentración de grelina circulante ${ }^{17}$.

Estos resultados sugieren que la incorporación de prebióticos a la dieta puede ser una estrategia interesante para controlar el apetito.

\section{La MI como un factor favorecedor del almacenamiento de grasa}

La hipótesis de que la MI podría actuar como un factor favorecedor del almacenamiento de grasa en el organismo surge de la observación que los ratones axénicos, a pesar de consumir $30 \%$ más alimento que los animales convencionales de la misma edad y peso, tienen $42 \%$ menos grasa corporal ${ }^{11}$. La convencionalización de los animales axénicos (proceso de transplante de la MI de ratones convencionales donantes al TD de ratones axénicos recipientes) produce en sólo 10 días un aumento de $57 \%$ de su grasa corporal total y una disminución correlativa de su consumo de alimentos (por lo cual su peso no se ve afectado $)^{11}$. Dicho fenómeno es acompañado por una elevación significativa de la glicemia, insulinemia y leptinemia y por un aumento de 2,3 veces del contenido hepático de triglicéridos, debido a la mayor expresión de la acetil-CoA carboxilasa y de la sintetasa de ácidos grasos. El transporte intestinal y la captación hepática de monosacáridos es dos veces mayor en los ratones convencionalizados que en los axénicos, debido en parte a la mayor vascularización de su mucosa intestinal ${ }^{18}$. A este fenómeno hay que agregar el rescate colónico de energía a partir de la fibra que genera AGV utilizados como substratos para la lipogenesis de novo a nivel hepático. Los adipocitos de los animales convencionalizados son de mayor tamaño que aquellos de los ratones axénicos. Esto se debería al hecho que la actividad de la lipoproteína lipasa (LPL), una enzima implicada en la captación celular de ácidos grasos a partir de las lipoproteínas y en la acumulación de triglicéridos en los adipocitos, aumenta de $122 \%$ en el tejido adiposo y $100 \%$ en el corazón de los animales convencionalizados ${ }^{11}$. Esta mayor actividad LPL se debe a la inhibición por la MI de la Fiaf ("FastingInduced Adipocyte Factor"), una hormona expresada constitutivamente en la mucosa intestinal de los ratones axénicos y que actúa como inhibidor circulante de la LPL ${ }^{19}$. Este fenómeno explicaría el mayor almacenamiento de grasa en los animales con MI. La inhibición de la Fiaf por la MI es una respuesta evolutivamente conservada puesto que también se observa durante la convencionalización del pez cebra ${ }^{20}$. La Fiaf sería, por lo tanto, un mediador clave en la capacidad de la MI de promover el almacenamiento de grasa en los adipocitos del huésped. Estudios más recientes confirman que la MI afecta tanto el metabolismo energético como lipídico del huésped ${ }^{21}$. Como consecuencia, los ratones axénicos se ven protegidos de la ganancia de peso cuando reciben por ocho semanas una dieta hipercalórica ${ }^{11}$. En efecto, al final de este período su peso es más de dos veces menor que el de los animales convencionalizados que reciben la misma dieta. Se observa también que dos horas después de la administración intragástrica de aceite de oliva, los niveles de triglicéridos circulantes bajan en los animales convencionales mientras permanecen elevados en los axénicos, debido a la inhibición de la LPL por la Fiaf circulante y, en consecuencia, por la menor capacidad de almacenar triglicéridos en 
los adipocitos ${ }^{11}$. Los ratones axénicos alimentados con una dieta hipergrasa son resistentes al desarrollo de la obesidad debido a que tienen una mayor tasa de oxidación de ácidos grasos en su músculo esquelético, capacidad que se pierde después de la convencionalización ${ }^{22}$. La importancia de la interrelación MI/Fiaf es confirmada por el hecho que ratones axénicos knock-out para la Fiaf pierden su resistencia a la obesidad mientras que ratones convencionales transgénicos que hiper-expresan Fiaf son más resistentes a la obesidad ${ }^{11,22}$. Por otra parte, el Archaea Methanobrevibacter smithii (un microorganismo metanoproductor) consume el $\mathrm{H}_{2}$ (producido en el colon por las fermentaciones) para reducir el $\mathrm{CO}_{2}$ hacia metano $\left(\mathrm{CH}_{4}\right)$; la eliminación del $\mathrm{H}_{2}$ "desecho" reduce la presión intraluminal de gas, facilitando la expansión de las demás poblaciones bacterianas y volviendo aún más eficiente el proceso de almacenamiento de grasa $^{23}$.

Estos resultados sugieren que, en el marco de la coevolución $\mathrm{MI} /$ huésped, la estimulación del almacenamiento de energía como grasa pudo haber representado una ventaja selectiva para el ser humano, probablemente en épocas ancestrales cuando era más difícil conseguir alimentos y las hambrunas eran más frecuentes.

\section{Microbiota y obesidad}

El análisis de la MI de ratones obesos ob/ob (causada por una mutación del gen de la leptina) muestra que las concentraciones colónicas de Firmicutes aumentan más de 50\% mientras que las de Bacteroidetes disminuyen correlativamente, comparado con los ratones normopeso ${ }^{24}$. A pesar de que las concentraciones de AGV en el ciego de los ratones obesos son mayores que en los normopeso, la cantidad de energía excretada en sus deposiciones es menor, indicando que el proceso de extracción y absorción de energía a partir de los alimentos es más eficiente en el animal obeso ${ }^{25}$. Esto se explica por el hecho que el número de genes dedicados a la hidrólisis de polisacáridos es mucho mayor en la comunidad bacteriana de los animales obesos que en la de los normopeso. Cabe destacar que los ratones axénicos normopesos que son convencionalizados con la MI de ratones obesos almacenan más grasa corporal y ganan más peso que aquellos animales convencionalizados con la
MI de ratones normopeso ${ }^{25}$. Estas observaciones indican que la mayor capacidad de extracción de energía y de almacenamiento de grasa es una característica transmisible en la cual participa la microbiota. La MI del ser humano obeso se comporta de la misma forma que la del ratón obeso: el porcentaje de Firmicutes es mayor y el de Bacteroidetes menor, en comparación con los sujetos normopeso ${ }^{24}$. El seguimiento de la MI de voluntarios obesos quienes recibieron una dieta hipocalórica durante un año muestra que a medida que los sujetos pierden peso, la proporción de Firmicutes disminuye mientras que aumenta la de Bacteroidetes ${ }^{26}$.

Estas múltiples observaciones, realizadas principalmente en sofisticados modelos animales, han gatillado en los últimos años el desarrollo de numerosos estudios para tratar de dilucidar la interrelación entre MI y obesidad en el ser humano. La Tabla 1 describe siete estudios que caracterizaron la MI de sujetos con sobrepeso y obesos ( 3 en adultos, 3 en niños o adolescentes y uno en mujeres embarazadas) en comparación con sujetos normopesos (y con sujetos anoréxicos en 1 estudio $)^{27-33}$. Si bien estos estudios confirman la existencia de alteraciones de la MI de los obesos, existen diferencias importantes en las poblaciones bacterianas involucradas debidas probablemente a las características de los sujetos reclutados, su dieta, la metodología utilizada y/o al diseño del estudio.

\section{Microbiota, dieta alta en grasa y estado inflamatorio}

En oposición con la hipótesis que relaciona obesidad con cambios de MI, Hildebrandt et $\mathrm{al}^{34}$ y anteriormente Cani et $\mathrm{a}^{35}$ han propuesto que dichos cambios podrían ser explicados por la mayor cantidad de grasa que llegan al colon. Los primeros utilizaron como modelo ratones knockout para RELM- $ß$ (Resistin-like molecule- $\beta$, una molécula expresada específicamente por las células caliciformes del epitelio colónico), los cuales muestran una incidencia menor de obesidad que los ratones silvestres alimentados con una dieta alta en grasa. Dicha dieta, efectivamente, disminuye los Bacteroidetes y aumenta correlativamente a Firmicutes y Proteobacteria, independientemente del fenotipo obeso o no-obeso de los animales, lo que sugiere que el contenido en grasa de la dieta, 
Tabla 1. Estudios de la microbiota intestinal de sujetos humanos obesos o con sobrepeso en comparación con individuos normopesos

\begin{tabular}{|c|c|c|c|c|}
\hline Sujetos & Tratamiento & Métodos & Resultados & $\begin{array}{l}\text { Refe- } \\
\text { rencias }\end{array}$ \\
\hline $\begin{array}{l}20 \text { obesos }+ \\
9 \text { anoréxicos }+ \\
20 \text { normopesos }\end{array}$ & $\operatorname{Sin}$ & $\begin{array}{l}\text { RT-PCR para determinar } \\
\text { las concentraciones de } \\
\text { Bacteroidetes, Firmicutes, } \\
\text { Lactobacillus y } M \text {. smithii }\end{array}$ & $\begin{array}{l}\downarrow \text { Bacteroidetes y } \uparrow \text { Lactobacillus } \\
\text { en obesos } \\
\uparrow M \text {. smithii en anoréxicos }\end{array}$ & 27 \\
\hline $\begin{array}{l}3 \text { normopesos } \\
+3 \text { obesos }+ \\
3 \text { post-by-pass } \\
\text { gástrico }\end{array}$ & $\operatorname{Sin}$ & $\begin{array}{l}\text { PCR-pyro-secuenciación y } \\
\text { análisis de } 184.094 \text { secuen- } \\
\text { cias de } 16 \text { sRNA }\end{array}$ & $\begin{array}{l}\text { Firmicutes predominantes en } \\
\text { normopesos y obesos pero } \downarrow \text { post } \\
\text { bypass gástrico } \\
\text { Gammaproteobacteria } \uparrow \text { post } \\
\text { bypass gástrico } \\
\text { M. smithii } \uparrow \text { en obesos }\end{array}$ & 28 \\
\hline $\begin{array}{l}30 \text { normopesos } \\
+35 \text { con } \\
\text { sobrepeso } \\
+33 \text { obesos }\end{array}$ & $\operatorname{Sin}$ & RT-PCR & $\begin{array}{l}\text { A mayor IMC, mayor concentra- } \\
\text { ción fecal de AGVs, la relación } \\
\text { Firmicutes/Bacteroidetes } \downarrow \text {, } \\
\text { Bifidobacterium } \downarrow \text { y } M \text {. smithii } \downarrow\end{array}$ & 29 \\
\hline $\begin{array}{l}36 \text { adolescentes } \\
\text { con sobrepeso }\end{array}$ & $\begin{array}{l}\text { Restricción calórica } \\
+ \text { actividad física } \\
\text { por } 10 \text { semanas }\end{array}$ & RT-PCR & $\begin{array}{l}\text { Con la baja de peso, Bacteroides } \\
\text { fragilis y Lactobacillus } \uparrow \text { mientras } \\
\text { Clostridium cocoides, B. longum y } \\
\text { B. adolescentis } \downarrow\end{array}$ & 30 \\
\hline $\begin{array}{l}13 \text { niños } \\
\text { normopesos y } \\
15 \text { obesos } \\
\text { (11-14 años) }\end{array}$ & $\operatorname{Sin}$ & RT-PCR & $\begin{array}{l}\text { Sin diferencia en Bacteroides, } \\
\text { Bifidobacterium, L. acidophillus y } \\
\text { E. rectale } \\
\uparrow \text { Faealibacterium prausnitzii }\end{array}$ & 31 \\
\hline $\begin{array}{l}18 \text { mujeres con } \\
\text { sobrepeso }+ \\
30 \text { normopesos, } \\
\text { todas } \\
\text { embarazadas }\end{array}$ & $\begin{array}{l}\text { Seguimiento } \\
\text { durante el } \\
\text { embarazo }\end{array}$ & $\begin{array}{l}\text { FISH-citometría de flujo + } \\
\text { RT-PCR }\end{array}$ & $\begin{array}{l}\uparrow \text { Bacteroides y Staphylococcus } \\
\text { con ganancia de peso excesiva }\end{array}$ & 32 \\
\hline $\begin{array}{l}25 \text { niños con } \\
\text { sobrepeso o } \\
\text { obesos }+24 \\
\text { niños normope- } \\
\text { sos (todos de } 7 \\
\text { años) }\end{array}$ & $\begin{array}{l}\text { Estudio } \\
\text { retrospectivo }\end{array}$ & $\begin{array}{l}\text { FISH-citometría de flujo + } \\
\text { RT-PCR }\end{array}$ & $\begin{array}{l}\text { Bifidobacterium mayor en niño } \\
\text { que permanecen normopesos } \\
\text { Staphylococus aureus mayor en } \\
\text { niños que vuelven obesos }\end{array}$ & 33 \\
\hline
\end{tabular}

y no el fenotipo obeso del individuo, es el factor determinante en los cambios en la MI observado en el sujeto obeso ${ }^{34}$. Tomando en cuenta que la RELM- $\beta$ ha sido propuesto como un potencial marcador de resistencia insulinica debido a su capacidad de inhibir las vías de señalización celular inducidas por insulina, es interesante notar que la expresión de RELM-ß en el colon es mayor en los ratones convencionalizados comparados con los ratones axénicos y que las concentraciones fecales y plasmáticas de esta molécula también son incrementadas por dieta alta en grasa ${ }^{36,37}$.
El impacto del contenido en grasa de la dieta sobre la MI y el desarrollo de inflamación de bajo grado ha sido estudiado en profundidad por Cani et al. Estos autores demostraron que la administración de una dieta alta en grasas por 4 semanas, además de alterar la MI de los ratones aumentando las bacterias gram-negativas a expensa de las gram-positivas, también afecta su función intestinal de barrera, inhibiendo la expresión de proteínas constitutivas de las uniones estrechas que unen a las células epiteliales entre $\mathrm{si}^{38}$. Este fenómeno tiene por consecuencia el incremento 
en 2-3 veces de las concentraciones plasmáticas de LPS que, por lo tanto, genera una endotoxinemia metabólica. Estos animales muestran además un incremento de su glicemia e insulinemia en ayunas y un aumento de su peso corporal y de su masa grasa y hepática mientras los niveles de citoquinas proinflamatorias (TNF- $\alpha$, IL- 6, IL- $1 \beta$, MIP-1) aumentan tanto en el plasma como en los tejidos adiposo, hepático y muscular ${ }^{38}$. Gran parte de estas alteraciones no son detectadas al administrar antibióticos a los animales, o al utilizar ratones "knockout" para el CD14 (el receptor de LPS), mientras son reproducidas por la infusión subcutánea crónica de LPS ${ }^{35,38}$. Estos hallazgos confirman, por lo tanto, el papel de la MI y en particular de las bacterias gram-negativas y de su LPS en el desarrollo del estado de inflamación y de la resistencia insulinica. En forma interesante, la administración dietaria de un prebiótico (la oligofructosa que estimula el crecimiento de las poblaciones gram-positivas Bifidobacterium y Lactobacillus en el colon) en ratones obesos ob/ ob protege frente a la alteración de permeabilidad intestinal, disminuye la endotoxinemia y el subsecuente aumento de marcadores plasmáticos y tisulares de estrés oxidativo y de inflamación ${ }^{39}$. Este efecto protector del prebiótico sobre la función intestinal de barrera es probablemente indirecto, mediado por la generación de AGV durante su fermentación, y la posterior estimulación de la liberación de GLP-2, una hormona digestiva con actividad trófica para la mucosa intestinal, por las células enteroendocrinas del epitelio intestinal ${ }^{40}$.

Corroborando los hallazgos obtenidos en modelos animales, se ha observado que la ingesta de una comida con alto contenido de grasa en sujetos sanos aumenta las concentraciones plasmáticas de LPS, contribuyendo al desarrollo de un estado inflamatorio post-prandial y a la activación de las células endoteliales ${ }^{41}$. En forma similar, Amar et al, observaron una correlación entre endotoxinemia y el consumo de energía y de grasa en un estudio realizado en 200 varones. La eventual alteración de la función intestinal de barrera como factor favorecedor del paso de LPS no fue evaluada en estos estudios. En un estudio reciente realizado en obesos jóvenes y asintomáticos, no observamos que existiera una asociación entre la alteración de la MI, la inflamación de la mucosa colónica y los cambios en la función intestinal de barrera (enviado a publicación).
Estos resultados sugieren que la disminución de los niveles plasmáticos de LPS podría ser una estrategia interesante para controlar los procesos inflamatorios asociados con las enfermedades metabólicas.

\section{Conclusiones}

Estudios realizados en los seis últimos años indican que la MI es un actor importante en la regulación del metabolismo energético del organismo. Además de su papel en el rescate colónico de energía, participa en el almacenamiento de grasa en los adipocitos. La MI de los obesos está alterada, comparada con aquella de los normopeso, lo que podría explicar su mayor eficiencia en la extracción de energía a partir de los alimentos. El contenido en grasa de la dieta también es un factor que puede alterar la composición de la MI, a través del aumento de las concentraciones plasmáticas de LPS y el consiguiente desarrollo de un estado proinflamatorio que facilita la aparición de resistencia insulinica. El consumo de prebióticos o de probióticos podría ayudar a mantener la homeostasis de la MI, previniendo las alteraciones anteriormente descritas y estimulando mecanismos implicados en la sensación de saciedad.

\section{Referencias}

1. Hooper LV, Wong MH, Thelin A, Hansson L, Falk PG, Gordon JI. Molecular analysis of commensal hostmicrobial relationships in the intestine. Science 2001; 291: 881-4.

2. Cummings JH, Pomare EW, Branch WJ, Naylor CP, Macfarlane GT. Short chain fatty acids in human large intestine, portal, hepatic and venous blood. Gut 1987; 28: 1221-7.

3. Dalby AB, Frank DN, St Amand AL, Bendele AM, Pace NR. Culture-independent analysis of indomethacin induced alterations in the rat gastrointestinal microbiota. Appl Environ Microbiol 2006; 72: 6707-15.

4. Brunser O, Gotteland M, Cruchet S, Figueroa G, Garrido D, Steenhout P. Effect of a milk formula with prebiotics on the intestinal microbiota of infants after an antibiotic treatment. Pediatr Res 2006; 59: 451-6.

5. Hookman P, Barkin JS. Clostridium difficile-associated disorders/diarrhea and Clostridium difficile colitis: the emergence of a more virulent era. Dig Dis Sci 2007; 52: 1071-5. 
La microbiota intestinal: Un nuevo actor en el desarrollo de la obesidad - P. Morales et al

6. Neish AS. Microbes in gastrointestinal health and disease. Gastroenterology 2009; 136: 65-80.

7. Turner JR. Intestinal mucosal barrier function in health and disease. Nat Rev Immunol 2009; 9: 799-809.

8. Brunser O, Gotteland M. Prebiotics and probiotics in human health: an overview. In Press in: "Bioactive foods in health promotion: probiotics and prebiotic." Watson $\mathrm{R}$ and Preedy V, Ed. Academic press, Kidlingto, UK.

9. Sonnenburg ED, Sonnenburg JL, Manchester JK, Hansen EE, Chiang HC, Gordon JI. A hybrid two component system protein of a prominent human gut symbiont couples glycan sensing in vivo to carbohydrate metabolism. Proc Natl Acad Sci USA 2006; 103: 8834-9.

10. Wong JMW, de Souza R, Kendall CW, Emam A, Jenkins DJA. Colonic health: fermentation and short chain fatty acids. J Clin Gastroenterol 2006, 40: 235-43.

11. Backhed F, Ding H, Wang T, Hooper LV, Koh GY, Nagy A, et al. The gut microbiota as an environmental factor that regulates fat storage. Proc Natl Acad Sci USA 2004; 101: 15718-23.

12. McNeil NI. The contribution of the large intestine to energy supplies in man. Am J Clin Nutr 1984; 39: 33842.

13. Delzenne NM, Cani PD, Daubioul C, Neyrinck AM. Impact of inulin and oligofructose on gastrointestinal peptides. Br J Nutr 2005; 93 Suppl 1: S157-61.

14. Cani PD, Hoste S, Guiot Y, Delzenne NM. Dietary nondigestible carbohydrates promote L-cell differentiation in the proximal colon of rats. Br J Nutr 2007; 98: 32-7.

15. Cani PD, Neyrinck AM, Maton N, Delzenne NM. Oligofructose promotes satiety in rats fed a high-fat diet: involvement of Glucagon-Like Peptide-1. Obes Res 2005; 13: 1000-7.

16. Cani PD, Lecourt E, Dewulf EM, Sohet FM, Pachikian BD, Naslain D, et al. Gut microbiota fermentation of prebiotics increases satietogenic and incretin gut peptide production with consequences for appetite sensation and glucose response after a meal. Am J Clin Nutr 2009; 90: 1236-43.

17. Parnell JA, Reimer RA. Weight loss during oligofructose supplementation is associated with decreased ghrelin and increased peptide YY in overweight and obese adults. Am J Clin Nutr 2009; 89: 1751-9.

18. Stappenbeck TS, Hooper LV, Gordon JI. Developmental regulation of intestinal angiogenesis by indigenous microbes via Paneth cells. Proc Natl Acad Sci USA 2002; 99: 15451-5.

19. Mandard S, Zandbergen F, van Straten E, Wahli W, Kuipers F, Muller M, et al. The fasting-induced adipose factor/angiopoietin-like protein 4 is physically associated with lipoproteins and governs plasma lipid levels and adiposity. J Biol Chem 2006; 281: 934-44.

20. Rawls JF, Samuel BS, Gordon JI. Gnotobiotic zebrafish reveal evolutionarily conserved responses to the gut microbiota. Proc Natl Acad Sci USA 2004; 101: 4596-601.

21. Velagapudi VR, Hezaveh R, Reigstad CS, Gopalacharyulu PV, Yetukuri L, Islam S, et al. The gut microbiota modulates host energy and lipid metabolism in mice. J Lipid Res 2010; 51: 1101-12.

22. Backhed F, Manchester JK, Semenkovich CF, Gordon JI. Mechanisms underlying the resistance to diet induced obesity in germ-free mice. Proc Natl Acad Sci USA 2007; 104: 979-84.

23. Samuel BS, Gordon JI. A humanized gnotobiotic mouse model of host-archaeal-bacterial mutualism. Proc Natl Acad Sci USA 2006; 103: 10011-6.

24. Ley RE, Backhed F, Turnbaugh P, Lozupone CA, Knight RD, Gordon JI. Obesity alters gut microbial ecology. Proc Natl Acad Sci USA 2005; 102: 11070-5.

25. Turnbaugh PJ, Ley RE, Mahowald MA, Magrini V, Mardis ER, Gordon JI. An obesity-associated gut microbiome with increased capacity for energy harvest. Nature 2006; 444: 1027-31

26. Ley RE, Turnbaugh PJ, Klein K, Gordon JI. Human gut microbes associated with obesity. Nature 2006; 444: 21 8.

27. Armougom F, Henry M, Vialettes B, Raccah D, Raoult D. Monitoring bacterial community of human gut microbiota reveals an increase in Lactobacillus in obese patients and Methanogens in anorexic patients. PLoS One 2009; 4: e7125.

28. Zhang H, DiBaise JK, Zuccolo A, Kudrna D, Braidotti $\mathrm{M}$, Yu Y, et al. Human gut microbiota in obesity and after gastric bypass. Proc Natl Acad Sci U S A 2009; 106: 2365-70.

29. Schwiertz A, Taras D, Schäfer K, Beijer S, Bos NA, Donus C, Hardt PD. Microbiota and SCFA in lean and overweight healthy subjects. Obesity 2010; 18: 190-5.

30. Santacruz A, Marcos A, Wärnberg J, Martí A, MartinMatillas M, Campoy C, et al. Interplay between weight loss and gut microbiota composition in overweight adolescents. Obesity 2009; 17: 1906-15.

31. Balamurugan R, George G, Kabeerdoss J, Hepsiba J, Chandragunasekaran AM, Ramakrishna BS. Quantitative differences in intestinal Faecalibacterium prausnitzii in obese Indian children. Br J Nutr 2009: 1-4.

32. Collado MC, Isolauri E, Laitinen K, Salminen S. Distinct composition of gut microbiota during pregnancy in overweight and normal-weight women. Am J Clin Nutr 2008; 88: 894-9.

33. Kalliomäki M, Collado MC, Salminen S, Isolauri E. Early differences in fecal microbiota composition in children 
may predict overweight. Am J Clin Nutr 2008; 87: 534-8.

34. Hildebrandt MA, Hoffmann C, Sherrill-Mix SA, Keilbaugh SA, Hamady M, Chen YY, Knight R, et al. Highfat diet determines the composition of the murine gut microbiome independently of obesity. Gastroenterology 2009; 137: 1716-24.

35. Cani PD, Amar J, Iglesias MA, Poggi M, Knauf C, Bastelica D, et al. Metabolic endotoxemia initiates obesity and insulin resistance. Diabetes 2007; 56: 1761-72.

36. Shojima N, Ogihara T, Inukai K, Fujishiro M, Sakoda H, Kushiyama A, et al. Serum concentrations of resistin-like molecules beta and gamma are elevated in high-fat-fed and obese $\mathrm{db} / \mathrm{db}$ mice, with increased production in the intestinal tract and bone marrow. Diabetologia 2005; 48: 984-92.

37. He W, Wang ML, Jiang HQ, Steppan CM, Shin ME, Thurnheer MC, et al. Bacterial colonization leads to the colonic secretion of RELMbeta/FIZZ2, a novel goblet cellspecific protein. Gastroenterology 2003; 125: 1388-97.

38. Cani PD, Bibiloni R, Knauf C, Waget A, Neyrinck AM, Delzenne1 NM, et al. Changes in gut microbiota control metabolic endotoxemia-induced inflammation in highfat diet-induced obesity and diabetes in mice. Diabetes 2008; 57: 1470-81.

39. Cani PD, Neyrinck AM, Fava F, Knauf C, Burcelin RG, Tuohy KM, et al. Selective increases of bifidobacteria in gut microflora improve high-fat-diet-induced diabetes in mice through a mechanism associated with endotoxinaemia. Diabetologia 2007; 50: 2374-83.

40. Cani PD, Possemiers S, Van de Wiele T, Guiot Y, Everard A, Rottier O, et al. Changes in gut microbiota control inflammation in obese mice through a mechanism involving GLP-2 driven improvement of gut permeability. Gut 2009; 58: 1091-103.

41. Erridge C, Attina T, Spickett CM, Webb DJ. A high-fat meal induces low-grade endotoxemia: evidence of a novel mechanism of postprandial inflammation. Am J Clin Nutr 2007; 86: 1286-92.

42. Amar J, Burcelin R, Ruidavets JB, Cani PD, Fauvel J, Alessi MC, et al. Energy intake is associated with endotoxemia in apparently healthy men. Am J Clin Nutr 2008; 87: 1219-23. 